\title{
ON THE CONSTRUCTION OF RELATED DIFFERENTIAL EQUATIONS $\left({ }^{1}\right)$
}

\author{
BY \\ RUDOLPH E. LANGER
}

1. Introduction. Many ordinary linear differential equations that are important in pure and in applied mathematics are of the type

$$
\frac{d^{n} u}{d z^{n}}+\lambda p_{1}(z, \lambda) \frac{d^{n-1} u}{d z^{n-1}}+\cdots+\lambda^{n} p_{n}(z, \lambda) u=0,
$$

in which $\lambda$ is a parameter whose absolute value is large, and the coefficients $p_{i}(z, \lambda)$ are either functions of $z$ alone, or series in powers of $1 / \lambda$ with coefficients that are functions of $z$. The forms of the solutions of such an equation, as they depend asymptotically upon $\lambda$ in a given region of the variable $z$, are known to be determined in large measure, though not entirely, by the configuration of the roots of the auxiliary algebraic equation

$$
\chi^{n}+p_{1}(z, \infty) \chi^{n-1}+\cdots+p_{n}(z, \infty)=0,
$$

in the given $z$-region. If the roots are all simple (distinct) for every $z$, the asympototic forms of the solutions are derivable by well established theory [1]. The same is true if some roots are multiple, but every multiplicity among them maintains identically over the region [2]. The cases for which relatively complete theory exists are therefore those in which the auxiliary root configuration is of an invariant kind.

More recent investigations $[3 ; 4 ; 5 ; 6]$ of differential equations $(1.1)$ have been directed toward determining the forms of their solutions in $z$-regions over which the configuration of auxiliary roots is not invariant, but in which there is a point-a so-called turning point or transition point-at which the configuration is different than it is elsewhere. Among turning points the simpler ones are those in which just two roots that are otherwise simple coincide. Even these fall into different categories, depending both upon the number of other roots that are involved (namely the order of the differential equation), and upon the degree to which the discriminant of the auxiliary equation vanishes where the multiplicity of roots occurs. The solutions, at least in the case of differential equations of the second order, are known to have quite different forms when this degree is 1 and when it is 2 .

Presented to the Society, August 30, 1955; received by the editors October 5, 1955.

(1) This research was supported by the United States Air Force through the Office of Scientific Research, Air Research and Development Command. 
Differential equations of the second order with turning points are important in a number of applied fields, notably in quantum mechanics and the theory of micro-wave propagation [7]. Such equations of the fourth and sixth orders have roles in hydrodynamics, where they arise in connection with the phenomenon of turbulence. Far from being rare, turning points present themselves in connection with many of the standard differential equations, such as the equations of Bessel, Legendre, Mathieu, Hermite, Laguerre, Whittaker, etc.

Since, for a differential equation of the second order, there are only two auxiliary roots, a turning point in connection with such an equation simply marks the conjunction of these two roots. For differential equations of higher orders a variety of coincidence configurations is possible, the number of possibilities increasing rapidly with the order of the equation. For example, in the case of a differential equation of the third order the roots may be simple except at the turning point, with the multiplicity there involving either just two or all three of them. Or a root which is simple except at the turning point may coincide there with one that is identically double over the region. The fourth order differential equation of hydrodynamics is featured by a turning point in which two roots that are otherwise simple and one that is identically double over the region fall into a four-fold multiplicity.

If the number of auxiliary roots that are involved in coincidences at a turning point is $m$, we shall say that the coincidence pattern at the point is $m$-fold. For $m>2$ a variety of such patterns exists, for, as has already been remarked, the roots may fall into a single multiplicity in which they all coincide, or they may fall together in several separate sets in various ways. Any particular $m$-fold pattern may present itself in connection with a differential equation (1.1) of any order that is greater than or equal to $m$. If $n>m$ there is an excess of auxiliary roots to the number $(n-m)$ that remain simple. Any specific $m$-fold pattern therefore evidently presents itself in its simplest form in the instance of a differential equation of the order $m$, since there are then no additional roots to engender accompanying complexities.

A method that has been used with some success for deriving the forms of the solutions of a differential equation (1.1) in a region containing a turning point is that of "related equations." This method requires, in the first place, the construction of a differential equation-known as a related equationwhose solution forms are known, and which, at the same time, has coefficients that are identical with those of the given equation to the extent of all terms to some prescribed degree in $1 / \lambda$. From the known solution forms for this related equation the corresponding ones for the given equation are then deduced. The possibility of the construction of a related equation is thus a prerequisite for applicability of the method. Whether this construction can be made, and if so how it is to be made, in the instance of a given differential equation with a specific coincidence pattern, largely remains a matter for 
research. The construction has been given only in connection with a few of the simpler configurations. This is so, specifically, for equations of the second order whose auxiliary equations have discriminants that vanish to the first or second degree; for equations of the third order in which those same coincidence patterns are present; and for certain differential equations of the third and fourth orders with rather particular three-fold and four-fold coincidence patterns respectively.

The purpose of the present paper can now be explained. It is to show, for any differential equation (1.1) of the $n$th order with an $m$-fold coincidence pattern, $m<n$, that the construction of a related equation is possible-and how it is to be made-if such a construction is known for a differential equation of the lower order $m$ having the same coincidence pattern. In effect it therefore disposes of the complications that are generated by the presence of $(n-m)$ simple auxiliary roots, by referring the construction of a related equation to a corresponding case in which no such roots are present. This at once greatly widens the category of differential equations (1.1) for which related equations are constructible. For instance, the problem for any differential equation (1.1) whose coincidence pattern is two-fold is hereby referred to its counterpart for differential equations of the second order. The constructions that are known for the second order case when the discriminant of the auxiliary equation vanishes to the first or second degree are thus made available for equations of all orders. Similarly, in the case of those three-fold and fourfold coincidence patterns for which the construction of a related equation has been accomplished in connection with respective differential equations of the third and fourth orders, the constructions are hereby extended to equations of higher order. The extension is, in fact, given in advance, for all constructions that may in the future be discovered in connection with differential equations of specific orders.

2. A differential form $L(u)$ and its derivatives. With $D$ denoting the operator of differentiation, so that $D^{i} u$ signifies the $i$ th derivative of $u$, and $D^{0} u$ is $u$ itself, the given differential equation (1.1) can be written as

$$
\mathcal{L}(u)=0 \text {, }
$$

with

$$
\mathcal{L}(u) \equiv \sum_{i=0}^{n} \lambda^{i} p_{i}(z, \lambda) D^{n-i} u .
$$

With respect to this we assume that $\lambda$, which may be real or complex, is bounded from zero in absolute value, and is otherwise unbounded. The coefficients $p_{i}(z, \lambda)$ we take to be representable by power series in $1 / \lambda$. Thus

$$
\begin{aligned}
p_{0} & \equiv 1, \\
p_{i}(z, \lambda) & =\sum_{j=0}^{\infty} \frac{p_{i, j}(z)}{\lambda^{i}}, \quad i=1,2, \cdots, n,
\end{aligned}
$$


these several series being convergent when $|\lambda|$ is sufficiently large. As to the functions $p_{i, j}(z)$, we assume them to be analytic in the $z$-region in which the differential equation is being considered. This region we shall think of as bounded and closed, so that functions that are analytic in it are bounded. The phrase "for all $z$ " shall be understood to mean all $z$ in this region.

The auxiliary equation (1.2) is, therefore,

$$
\Omega(\chi)=0,
$$

with

$$
\Omega(\chi) \equiv \chi^{n}+p_{1,0}(z) \chi^{n-1}+\cdots+p_{n, 0}(z) .
$$

We suppose that within the z-region there is a turning point, at which coincidences not maintaining elsewhere occur among the roots of this equation in accordance with some $m$-fold pattern, with $m<n$. The $(n-m)$ roots, $\chi_{1}(z), \cdots, \chi_{n-m}(z)$, that are not involved in this pattern are taken to be distinct for all $z$, whereas the $m$ roots $\chi_{n-m+1}(z), \cdots, \chi_{n}(z)$, that are involved in the pattern are each either otherwise distinct or are part of an identical multiplicity over the region.

Let the integer $r$ be chosen arbitrarily, but, once chosen, let it be fixed. We shall be concerned with the construction of a related equation whose coefficients are the same as those of the given equation (1.1), to the extent of all terms of degree less than or equal to the $r$ th in $1 / \lambda$.

Consider a differential form

$$
L(u) \equiv \sum_{i=0}^{m} \lambda^{i} q_{i}(z, \lambda) D^{m-i} u,
$$

that conforms with the following description. Of its coefficients the leading one is 1 , and the others are expressible in powers of $1 / \lambda$, thus

$$
\begin{aligned}
q_{0} & \equiv 1, \\
q_{i}(z, \lambda) & =\sum_{j=0}^{\infty} \frac{q_{i, j}(z)}{\lambda^{j}}, \quad i=1,2, \cdots, m .
\end{aligned}
$$

The functions $q_{i, j}(z)$ are to be analytic for all $z$. Those with subscripts $j \leqq r$ are to be, for the moment, undetermined, and are to be subject to later specifications. Those with subscripts $j>r$ may be specifically assigned at once any values for which the series (2.7) converge. A particular assignment that may be made is $q_{i, j} \equiv 0$ for $j>r$, but this we do not insist upon.

The derivatives of the form (2.6) are given by the formula

$$
D^{k} L(u)=\sum_{i=0}^{m+k} \lambda^{i} Q_{i}^{(k)} D^{m+k-i} u, \quad k=1,2, \cdots,(n-m),
$$

in which the coefficients are 


$$
Q_{i}^{(k)}=\sum_{j=0}^{k}\left(\begin{array}{l}
k \\
j
\end{array}\right) \frac{D^{j} q_{i-j}}{\lambda^{i}}
$$

The symbol

$$
\left(\begin{array}{l}
k \\
j
\end{array}\right)
$$

designates, as usually, the coefficient of $x^{j}$ in the binomial expansion of $(1+x)^{k}$. It is to be understood, of course, that in any formula, $q_{i} \equiv 0$, for $i<0$, and $i>m$.

For many of the considerations that we shall have to make, the matters of essential moment will be the particular arguments from which certain functions are constructed, and the characters of the constructions. To indicate that, a generic notation is convenient. We shall therefore reserve the symbol $I$ with any set of analytic arguments $\alpha_{1}, \cdots, \alpha_{s}$, thus $I\left(\alpha_{1}, \cdots, \alpha_{s}\right)$, to signify a (some) function that can be generated solely by means of additions, multiplications and differentiations from constant multiples of the arguments indicated, possibly in conjunction with known functions (not indicated) that are analytic in $z$ and are power series in $1 / \lambda$. It will be clear that any function $I\left(\alpha_{1}, \cdots, \alpha_{s}\right)$ is analytic, and that the symbol for the function itself applies also to the derivative. Also any sum or product of functions $I$ is again such a function, the arguments of which include all those of the terms or factors. As is usual, of course, a function, though denoted by $I\left(\alpha_{1}, \cdots, \alpha_{s}\right)$, may, in fact, actually depend only upon a sub-set of the indicated arguments.

In terms of the notation thus described, it will be seen from (2.9) that

$$
\begin{array}{lrl}
Q_{0}^{(k)} \equiv 1, & \text { for } k=1,2, \cdots,(n-m), \\
Q_{i}^{(k)}=q_{i}+\frac{1}{\lambda} I\left(q_{1}, \cdots, q_{i-1}, \frac{1}{\lambda}\right), & i=1,2, \cdots, m, \\
Q_{i}^{(k)}=\frac{1}{\lambda} I\left(q_{1}, \cdots, q_{m}, \frac{1}{\lambda}\right), & i=m+1, \cdots, m+k, \\
Q_{i}^{(k)} \equiv 0, & \text { for } i<0, \text { and for } i>m+k .
\end{array}
$$

3. The differential operators $\mathcal{D}_{k}$. Let the functions of a set $\sigma_{1}(z, \lambda), \cdots$, $\sigma_{n-m}(z, \lambda)$ be expressible in powers of $1 / \lambda$, thus

$$
\sigma_{i}(z, \lambda)=\sum_{j=0}^{\infty} \frac{\sigma_{i, j}(z)}{\lambda^{j}}, \quad i=1,2, \cdots,(n-m),
$$

with coefficients $\sigma_{i, j}(z)$ that are analytic for all $z$. Of these coefficients, let those for which $j \leqq r$ be undetermined, whereas the remaining ones are given any values for which the several series (3.1) are convergent when $|\lambda|$ is 
sufficiently large. The particular assignment $\sigma_{i j} \equiv 0$ for $j>r$ is admissible. In terms of these functions we define the differential operators $\mathcal{D}_{\boldsymbol{k}}$ by the formulas

$$
\mathcal{D}_{k}=\prod_{i=1}^{k}\left(D-\lambda \sigma_{i}\right), \quad k=1,2, \cdots,(n-m),
$$

the order of the symbolic factors being that in which $i$ increases toward the left.

Every such operator is, of course, expressible as a polynomial in $D$, thus

$$
\mathcal{D}_{k}=\sum_{i=0}^{k} \lambda^{i} \theta_{i}^{(k)} D^{k-i}
$$

In fact on applying this form to the relation

$$
\mathscr{D}_{k}=\left(D-\lambda \sigma_{k}\right) \mathcal{D}_{k-1},
$$

it is found that the coefficients $\theta_{i}^{(k)}$ conform to the recurrence relations

$$
\begin{aligned}
\theta_{0}^{(k)}=1, & \text { for } k=1,2, \cdots,(n-m), \\
\theta_{i}^{(k)}=\theta_{i}^{(k-1)}-\sigma_{k} \theta_{i-1}^{(k-1)}+\frac{1}{\lambda} D \theta_{i-1}^{(k-1)}, & i=1,2, \cdots, k, \\
\theta_{i}^{(k)} \equiv 0, & \text { for } i<0, \text { and for } i>k .
\end{aligned}
$$

From these the functions $\theta_{i}^{(k)}$ would be obtainable. We merely observe, however, that $\theta_{i}^{(k)}$ is constructed of only those functions $\sigma_{j}$ for which $j \leqq k$, namely that

$$
\theta_{i}^{(k)}=I\left(\sigma_{1}, \cdots, \sigma_{k}, \frac{1}{\lambda}\right) .
$$

The result of the direct application of the operator $\mathcal{D}_{k}$ to the form $L(u)$ may be taken from the relations (3.3) and (2.8) to be the formula

$$
\mathcal{D}_{k} L(u)=\sum_{i=0}^{k} \sum_{j=0}^{m+k-i} \lambda^{i+j} \theta_{i}^{(k)} Q_{j}^{(k-i)} D^{m+k-i-j} u .
$$

To rearrange the terms of this into an order of descending powers of $D$, we begin by replacing the upper summation limit on $i$ by $m+k$. This is permissible, because the terms that are thereby formally added each contain a factor $\theta_{i}^{(k)}$ which vanishes for the index $i$ in question. If a new summation index $l$ is then introduced to replace $j$ by virtue of the relation $i+j=l$, and the order of summation is thereafter interchanged, the resulting form (with $i$ and $j$ written again in the places of $l$ and $i$ ) is 


$$
\Phi_{k} L(u)=\sum_{i=0}^{m+k} \lambda^{i} \tau_{i}^{(k)} D^{m+k-i} u
$$

with

$$
\tau_{i}^{(k)}=\sum_{j=0}^{i} \theta_{i}^{(k)} Q_{i-i}^{(k-j)}
$$

In the initial term of the formula (3.8) the factor $Q_{i}^{(k)}$ is, by (2.10), of the form $q_{i}+(1 / \lambda) I\left(q_{1}, \cdots, q_{i-1}, 1 / \lambda\right)$, and its multiplier $\theta_{0}^{(k)}$ is 1 . In each subsequent term the factor $Q_{i-j}^{(\boldsymbol{k}-j)}$ depends only upon functions $q_{h}$ for which $h \leqq i-1$, and its multiplier is of the form (3.6). We see thus that

$$
\tau_{i}^{(k)}=q_{i}+I\left(q_{1}, \cdots, q_{i-1}, \sigma_{1}, \cdots \sigma_{k}, \frac{1}{\lambda}\right) .
$$

Now since each function $q_{i}$ and $\sigma_{i}$ is expressible as a power series in $1 / \lambda$, the same is true of any function $I$ with these arguments. It is, in fact, readily seen that

$$
I\left(q_{1}, \cdots, q_{h}, \sigma_{1}, \cdots, \sigma_{k}, \frac{1}{\lambda}\right)=\sum_{j=0}^{\infty} \frac{I\left(q_{1,0}, \cdots, q_{h, j}, \sigma_{1,0}, \cdots \sigma_{k, j}\right)}{\lambda^{i}},
$$

it being an important feature that the coefficient of $1 / \lambda^{j}$ on the right involves the functions $q_{i, h}$ and $\sigma_{i, h}$ only for $h \leqq j$. Thus, by (3.9)

$$
\tau_{i}^{(k)}=\sum_{j=0}^{\infty} \frac{1}{\lambda^{i}}\left\{q_{i, j}+I\left(q_{1,0}, \cdots, q_{i-1, j}, \sigma_{1,0}, \cdots, \sigma_{k, j}\right)\right\} .
$$

The relations (2.2) and (3.7) permit us to write

$$
\mathcal{L}(u)-\mathcal{D}_{n-m} L(u)=\sum_{i=0}^{n} \lambda^{i}{ }_{\epsilon_{i}} D^{n-i} u
$$

with

$$
\epsilon_{0}=0, \epsilon_{i}=p_{i}-\tau_{i}^{(n-m)}, \quad i=1,2, \cdots, n .
$$

4. Some formal evaluations. If the relations (3.12) are respectively multiplied by $\omega^{n-i}$, where $\omega$ is any function of the form

$$
\omega=\sum_{j=0}^{\infty} \frac{\omega_{j}(z)}{\lambda^{i}}
$$

their resultant sum is

$$
\sum_{i=0}^{n} \epsilon_{i} \omega^{n-i}=E_{1}(\omega)-E_{2}(\omega)
$$


with

$$
\begin{aligned}
& E_{1}(\omega)=\sum_{i=0}^{n} p_{i} \omega^{n-i}, \\
& E_{2}(\omega)=\sum_{i=0}^{n} \tau_{i}^{(n-m)} \omega^{n-i} .
\end{aligned}
$$

We need to analyse each of the functions (4.3) as a power series in $1 / \lambda$.

From the formula (4.1) it follows that for any integral exponent $h$

$$
\omega^{h}=\omega_{0}^{h}+\sum_{j=1}^{\infty} \frac{h \omega_{0}^{h-1} \omega_{j}+I\left(\omega_{0}, \cdots, \omega_{j-1}\right)}{\lambda^{j}} .
$$

This can be proved by induction. Since the functions $p_{i, j}(z)$ are known, and therefore need not be indicated as arguments in a function $I$, it follows that

$$
p_{i} \omega^{n-i}=p_{i, 0}^{n-i}+\sum_{j=1}^{\infty} \frac{(n-i) p_{i 0} \omega_{0}^{n-i-1} \omega_{j}+I\left(\omega_{0}, \cdots, \omega_{j-1}\right)}{\lambda^{i}} .
$$

Accordingly

$$
E_{1}(\omega)=\Omega\left(\omega_{0}\right)+\sum_{j=1}^{\infty} \frac{\dot{\Omega}\left(\omega_{0}\right) \omega_{j}+I\left(\omega_{0}, \cdots \omega_{j-1}\right)}{\lambda^{i}},
$$

where $\Omega(\chi)$ has been given in (2.5), and

$$
\dot{\Omega}(\chi)=n \chi^{n-1}+(n-1) p_{1,0}(z) \chi^{n-2}+\cdots+p_{n-1,0}(z) .
$$

Consider now the function $E_{2}(\omega)$. By (4.3) and (3.8) the formula for this is

$$
E_{2}(\omega)=\sum_{i=0}^{n} \sum_{j=0}^{i} \theta_{j}^{(n-m)} Q_{i-j}^{(n-m-j)} \omega^{n-i} \text {. }
$$

An interchange of the order of summation, followed by the introduction of a new summation index $l$ to replace $i$ by virtue of the relation $i=l+j$, gives it the form

$$
E_{2}(\omega)=\sum_{j=0}^{n} \sum_{l=0}^{n-j} \theta_{j}^{(n-m)} Q_{l}^{(n-m-j)} \omega^{n-j-l} .
$$

In this the upper summation limit upon $j$ can be reduced to $(n-m)$, since the terms that are thereby formally omitted contain factors $\theta_{j}^{(n-m)}$ which vanish for these indices $j$. By use of the evaluations (2.10) it may then be seen that

$$
E_{2}(\omega)=\sum_{j=0}^{n-m} \sum_{l=0}^{m} \theta_{j}^{(n-m)} q_{l} \omega^{n-i-l}+\frac{1}{\lambda} \sum_{j=0}^{n-m} \sum_{l=0}^{n-1} \theta_{j}^{(n-m)} I\left(q_{1}, \cdots, q_{l-1}, \frac{1}{\lambda}\right) \omega^{n-j-l} .
$$


The first double sum in this formula is factorable. We see thus that

$$
E_{2}(\omega)=S_{n-m} \sum_{l=0}^{m} q_{l} \omega^{m-l}+\frac{1}{\lambda} I\left(q_{1}, \cdots, q_{m}, \sigma_{1}, \cdots, \sigma_{n-m}, \omega, \frac{1}{\lambda}\right),
$$

where

$$
S_{k}(\omega)=\sum_{j=0}^{k} \theta_{j}^{(k)} \omega^{k-j} .
$$

We may obtain an evaluation of the factor $S_{n-m}(\omega)$ by suitably operating upon the function $\exp \left\{\lambda \int \omega d z\right\}$. Thus it follows readily that

$$
D^{i} \exp \left(\lambda \int \omega d z\right)=\exp \left(\lambda \int \omega d z\right)\left\{\lambda^{i} \omega^{j}+\lambda^{i-1} I\left(\omega, \frac{1}{\lambda}\right)\right\} .
$$

By (3.3), therefore,

$$
\mathcal{D}_{k} \exp \left(\lambda \int \omega d z\right)=\exp \left(\lambda \int \omega d z\right)\left\{\lambda^{k} S_{k}(\omega)+\lambda^{k-1} I\left(\theta_{1}^{(k)}, \cdots, \theta_{k}^{(k)}, \omega, \frac{1}{\lambda}\right)\right\} .
$$

This, however, implies that

$$
\begin{aligned}
(D & \left.-\lambda \sigma_{k}\right) \mathcal{D}_{k-1} \exp \left(\lambda \int \omega d z\right) \\
& =\exp \left(\lambda \int \omega d z\right)\left\{\lambda^{k}\left(\omega-\sigma_{k}\right) S_{k-1}(\omega)+\lambda^{k-1} I\left(\theta_{1}^{(k-1)}, \cdots \theta_{k-1}^{(k-1)}, \sigma_{k}, \omega, \frac{1}{\lambda}\right)\right\} .
\end{aligned}
$$

Because the left-hand members of the two latter relations are the same, by (3.4), it may be seen from the right-hand members that

$$
S_{k}(\omega)=\left(\omega-\sigma_{k}\right) S_{k-1}(\omega)+\frac{1}{\lambda} I\left(\sigma_{1}, \cdots, \sigma_{k}, \omega, \frac{1}{\lambda}\right) .
$$

Since $S_{1}(\omega)=\left(\omega-\sigma_{1}\right)$, it is provable by induction that

$$
S_{k}(\omega)=\prod_{i=1}^{k}\left(\omega-\sigma_{i}\right)+\frac{1}{\lambda} I\left(\sigma_{1}, \cdots, \sigma_{k}, \omega, \frac{1}{\lambda}\right) .
$$

With this evaluation (4.9), the formula (4.7) becomes

$$
\begin{aligned}
E_{2}(\omega)= & \prod_{i=1}^{n-m}\left(\omega-\sigma_{i}\right) \sum_{l=0}^{m} q_{l} \omega^{m-l} \\
& +\frac{1}{\lambda} I\left(q_{1}, \cdots, q_{m}, \sigma_{1}, \cdots, \sigma_{n-m}, \omega, \frac{1}{\lambda}\right) .
\end{aligned}
$$

Expressed in powers of $1 / \lambda$, this is 


$$
\begin{aligned}
E_{2}(\omega)= & \prod_{i=1}^{n-m}\left(\omega_{0}-\sigma_{i, 0}\right) \sum_{l=0}^{m} q_{l, 0} \omega_{0}^{m-l} \\
& +\sum_{j=1}^{\infty} \frac{I\left(q_{1,0}, \cdots, q_{m, j}, \sigma_{1,0}, \cdots, \sigma_{n-m, j}, \omega_{0}, \cdots, \omega_{j}\right)}{\lambda^{j}}
\end{aligned}
$$

5. Determination of the functions $q_{i}$ and $\sigma_{i}$. By virtue of the relations (2.3), (3.10) and (3.12), the coefficients $\epsilon_{i}$ in the equation (3.11) have the forms

$$
\begin{array}{r}
\epsilon_{i}(z, \lambda)=\sum_{j=0}^{\infty} \frac{1}{\lambda^{j}}\left\{p_{i, j}-q_{i, j}+I\left(q_{1,0}, \cdots, q_{i-1, j}, \sigma_{1,0}, \cdots, \sigma_{n-m, j}\right)\right\}, \\
i=1,2, \cdots, n .
\end{array}
$$

We propose to determine the functions $q_{i}$ and $\sigma_{i}$, namely the first $(r+1)$ hitherto undetermined coefficients of the formulas (2.7) and (3.1), in such a way as to reduce each function $\epsilon_{i}$ to be of the order of $1 / \lambda^{r+1}$. Insofar as the functions $q_{i}$ are concerned, this will be done indirectly. Their coefficients $q_{i, j}(z)$ will first be determined in terms of those of the functions $\sigma_{1, j}, \cdots$, $\sigma_{n-m, j}$. These latter will thereupon be determined, wherewith the actual determination of the functions $q_{i}$ will, of course, then also be accomplished.

Consider the coefficients of the terms in $1 / \lambda^{j}$ for $0,1,2, \cdots, r$, in the formula (5.1) for $\epsilon_{1}$. They are the left-hand members of respective equations

$$
p_{1, j}-q_{1, j}+I\left(\sigma_{1,0}, \cdots, \sigma_{n-m, j}\right)=0, \quad j=0,1,2, \cdots, r .
$$

We assign to the functions $q_{1, j}(z)$ the values which fulfill these equations. This determines them in terms of the functions $\sigma_{1,0}, \cdots, \sigma_{n-m, j}$. Suppose, now, that the functions $q_{h, j}(z)$ have been determined for all $h \leqq i-1$. The coefficients of the terms in $1 / \lambda^{i}$ in the formula (5.1) for $\epsilon_{i}$ are then the lefthand members of respective equations

$$
p_{i, j}-q_{i, j}+I\left(q_{1,0}, \cdots q_{i-1, j}, \sigma_{1,0}, \cdots, \sigma_{n-m, j}\right)=0, j=0,1,2, \cdots, r,
$$

and by assigning to the functions $q_{i, 1}, \cdots, q_{i, r}$ the values which fulfill these equations they are determined in terms of the $\sigma_{i, j}$. In this way the functions $q_{i, j}$ may be determined for $i=1,2, \cdots, m$, and as a result we have

$$
\epsilon_{i}(z, \lambda)=\frac{1}{\lambda^{r+1}} I\left(\sigma_{1}, \cdots, \sigma_{n-m}, \frac{1}{\lambda}\right), \quad i=1,2, \cdots, m .
$$

The first $m$ of the functions $\epsilon_{i}$ have thus been given the desired form. The remaining $(n-m)$ of them have still to be considered.

With any one of the functions $\sigma_{k}$ in the role of $\omega$, the indicated product in the formula (4.10) vanishes, and accordingly

$$
E_{2}^{\prime}\left(\sigma_{k}\right)=\frac{1}{\lambda} \sum_{j=0}^{\infty} \frac{I\left(\sigma_{1,0}, \cdots, \sigma_{n-m, j}\right)}{\lambda^{j}} .
$$


By virtue of the relations (4.2) and (4.5), therefore,

$$
\begin{aligned}
& \sum_{i=0}^{n} \epsilon_{i} \sigma_{k}^{n-i}=\Omega\left(\sigma_{k, 0}\right)+\sum_{j=1}^{\infty} \frac{\dot{\Omega}\left(\sigma_{k, 0}\right) \sigma_{k, j}+I\left(\sigma_{1,0}, \cdots, \sigma_{n-m, j-1}\right)}{\lambda^{j}}, \\
& k=1,2, \cdots,(n-m) .
\end{aligned}
$$

To make the leading terms in these formulas vanish, we choose each $\sigma_{k, 0}(z)$ to be the respective function $\chi_{k}(z)$, a simple root of the equation (2.4). The coefficients of the terms in $1 / \lambda^{j}$ are then the left-hand members of respective equations

$$
\dot{\Omega}\left(\chi_{k}\right) \sigma_{k, j}+I\left(\sigma_{1,0}, \cdots, \sigma_{n-m, j-1}\right)=0, \quad k=1,2, \cdots,(n-m) .
$$

Since the root $\chi_{k}(z)$ is simple, the relation $\dot{\Omega}\left(\chi_{k}\right) \neq 0$ is fulfilled for all $z$. Therefore, when the functions $\sigma_{k, h}$ for $h \leqq j-1$ have been determined, the equations (5.5) determine the functions $\sigma_{k, j}$. In this way the undetermined coefficients of the functions $\sigma_{i}(z, \lambda)$ may all be determined, and these functions, as well as the functions $q_{i}(z, \lambda)$, may henceforth be dealt with as known. The basis of the determination has been the reduction of the right-hand members of the formulas (5.4) to be of the order of $1 / \lambda^{r+1}$. With the use of $B(z, \lambda)$ as a generic symbol to signify a (some) function which is expressible as a power series in $1 / \lambda$ with coefficients that are analytic in $z$, we may therefore write

$$
\sum_{i=0}^{n} \epsilon_{i} \sigma_{k}^{n-i}=\frac{B(z, \lambda)}{\lambda^{r+1}}, \quad k=1,2, \cdots,(n-m) .
$$

Now because the $\sigma_{i}$ have been determined, the relation (5.3) shows that

$$
\epsilon_{i}(z, \lambda)=\frac{B(z, \lambda)}{\lambda^{r+1}},
$$

at least for $i=1,2, \cdots, m$. By the transposition of the first $(r+1)$ terms from the left of the equations (5.6) we therefore obtain the set of equations

$$
\sum_{i=m+1}^{n} \epsilon_{i} \sigma_{k}^{n-i}=\frac{B(z, \lambda)}{\lambda^{r+1}}, \quad k=1,2, \cdots,(n-m) .
$$

This is a linear algebraic system for the unknowns $\epsilon_{m+1}, \cdots, \epsilon_{n}$. The determinant of the system, namely $\left|\sigma_{i}^{n-m-j}(z, \lambda)\right|$, is expressible as a power series in $1 / \lambda$. The "constant" term of this series is the determinant $\left|\chi_{i}^{n-m-j}(z)\right|$, and this is different from zero for all $z$ because the roots $\chi_{i}(z)$ are distinct for $i=1,2, \cdots,(n-m)$. The system (5.8) is therefore solvable, at least when $|\lambda|$ is sufficiently large. Its solution establishes the relations (5.7) for the remaining values $i=m+1, \cdots, n$.

By the determinations that have been made the differential operator $\mathcal{D}_{n-m}$ and the differential form $L(u)$ have both been made specific. 
6. The differential equation $L^{*}(u)=0$. The differential equation

$$
L(u)=0
$$

is now one in which the coefficients are known. Its auxiliary equation is

$$
\Omega_{0}(\chi)=0,
$$

with

$$
\Omega_{0}(\chi)=\sum_{i=0}^{m} q_{i, 0}(z) \chi^{m-i} .
$$

We have the means for analysing this auxiliary equation at hand. In the relation (4.2), with any function $\chi(z)$ in the place of $\omega(z, \lambda)$, each member is expressible as a power series in $1 / \lambda$. For the left-hand member, the constant term of that series is zero, since each $\epsilon_{i}$ is of the order of $1 / \lambda^{r+1}$. The constant terms of $E_{1}(\chi)$ and $E_{2}(\chi)$ are therefore the same, namely, by (4.5) and (4.11), and the fact that $\sigma_{i, 0}=\chi_{i}(z)$,

$$
\Omega(\chi)=\prod_{i=1}^{n-m}\left(\chi-\chi_{i}\right) \sum_{l=0}^{m} q_{l, 0} \chi^{m-l} .
$$

Now the functions $\chi_{i}(z)$ are the roots of the equation (2.4), and hence $\Omega(\chi)$ is the product of all the factors $\left(\chi-\chi_{i}\right)$ for $i=1,2, \cdots, n$. We conclude, therefore, that

$$
\sum_{l=0}^{m} q_{l, 0} \chi^{m-l}=\prod_{i=n-m+1}^{n}\left(\chi-\chi_{i}\right) .
$$

This is to say, that those auxiliary roots of the differential equation (2.1) that are involved in that equation's coincidence pattern at the turning point under consideration are precisely the auxiliary roots of the differential equation (6.1). This latter is therefore an equation having the same coincidence pattern as the originally given differential equation. It is evidently of the minimum order $m$ in which this pattern can occur.

It is the purpose of this discussion to show how a related differential equation can be constructed for a given equation (2.1) if that can be done for the simpler equation (6.1). We now assume, therefore, that a differential equation

$$
L^{*}(u)=0
$$

whose solution forms are known, and those coefficients are identical with those of the equation (6.1) to the extent of all terms up to those of the $r$ th degree in $1 / \lambda$, is constructible.

From the formulas (3.7) and (3.10), the coefficients of the differencial forms $\mathcal{D}_{k} L^{*}(u)$ and $\mathscr{D}_{k} L(u)$ may be seen to be identical to the same extent in powers of $1 / \lambda$, as are those of $L^{*}(u)$ and $L(u)$. Therefore, in the relation 


$$
\mathcal{L}(u)-\mathcal{D}_{n-m} L^{*}(u)=\sum_{i=1}^{n} \lambda^{i} \epsilon_{i}^{*} D^{n-i} u,
$$

the left-hand member has coefficients that differ from those of the corresponding member of (3.11) only by terms that are of higher degree in $1 / \lambda$ than the $r$ th. The same therefore maintains as to the right-hand members, namely, by (5.7),

$$
\stackrel{\epsilon_{i}^{*}}{*}=\frac{B(z, \lambda)}{\lambda^{r+1}}, \quad i=1,2, \cdots, n .
$$

7. The functions $u_{1}(z, \lambda), \cdots, u_{n-m}(z, \lambda)$. Let the functions $u_{i}(z, \lambda)$ be taken to be of the forms

$$
u_{i}(z, \lambda)=\exp \left(\lambda \int \sigma_{i}(z, \lambda) d z\right) A_{i}(z, \lambda), \quad i=1,2, \cdots,(n-m),
$$

with each $A_{i}(z, \lambda)$ a power series in $1 / \lambda$, thus

$$
A_{i}(z, \lambda)=\sum_{j=0}^{\infty} \frac{\alpha_{i, j}(z)}{\lambda^{i}} .
$$

The coefficients $\alpha_{i, j}(z)$ are to be analytic, and those for which $j<r$ are to be left undetermined. Those for which $j \geqq r$ may be specified in any way that makes the several series (7.2) convergent. In particular, they may be assigned the value 0 .

The set of evaluations

$$
D^{k} u_{i}=\lambda^{k} \exp \left(\lambda \int \sigma_{i} d z\right)\left\{\sigma_{i}^{k} A_{i}+\frac{1}{\lambda} I\left(A_{i}, \frac{1}{\lambda}\right)\right\}
$$

are easily established by the method of induction. Since the leading term of the formula (3.1) is $\chi_{i}(z)$, it follows that

$$
\sigma_{i}^{k}=\chi_{i}^{k}+\frac{1}{\lambda} B(z, \lambda),
$$

and therefore that

$$
\stackrel{\sigma_{i}^{k} A_{i}}{=} \underset{\chi_{i} \alpha_{i, 0}}{k}+\sum_{j=1}^{\infty} \frac{\chi_{i}^{k} \alpha_{i, j}+I\left(\alpha_{i, 0}, \cdots, \alpha_{i, j-1}\right)}{\lambda^{j}} .
$$

In accordance with this, a more explicit form of (7.3) is

$$
D^{k} u_{i}=\lambda^{k} \exp \left(\lambda \int \sigma_{i} d z\right)\left\{\chi_{i}^{k} \alpha_{i, 0}+\sum_{j=1}^{\infty} \frac{\chi_{i}^{k} \alpha_{i, j}+I\left(\alpha_{i, 0}, \cdots, \alpha_{i, j-1}\right)}{\lambda^{j}}\right\}
$$

A consequent evaluation is 


$$
\begin{aligned}
L^{*}\left(u_{i}\right)= & \lambda^{m} \exp \left(\lambda \int \sigma_{i} d z\right)\left\{\Omega_{0}\left(\chi_{i}\right) \alpha_{i, 0}\right. \\
& \left.+\sum_{j=1}^{\infty} \frac{\Omega_{0}\left(\chi_{i}\right) \alpha_{i, j}+I\left(\alpha_{i, 0}, \cdots, \alpha_{i, j-1}\right)}{\lambda^{j}}\right\} .
\end{aligned}
$$

That $L\left(u_{i}\right)$ has the form of the right-hand member of this equation would be found from the formulas (2.6) and (7.4). That the evaluation (7.5) applies to $L^{*}\left(u_{i}\right)$ follows, therefore, from the fact that the coefficients of the latter differ from those of $L\left(u_{i}\right)$ only by known functions that are analytic in $z$ and of degrees higher than the $r$ th in $1 / \lambda$. By induction it can now be proved that

with

$$
\begin{aligned}
\mathcal{D}_{k} L^{*}\left(u_{i}\right)= & \lambda^{m+k} \exp \left(\lambda \int \sigma_{i} d z\right)\left\{\Omega_{k}\left(\chi_{i}\right) \alpha_{i, 0}\right. \\
& \left.+\sum_{j=1}^{\infty} \frac{\Omega_{k}\left(\chi_{i}\right) \alpha_{i, j}+I\left(\alpha_{i, 0}, \cdots, \alpha_{i, j-1}\right)}{\lambda^{i}}\right\}
\end{aligned}
$$

$$
\Omega_{k}(\chi)=\Omega_{0}(\chi) \prod_{j=1}^{k}\left(\chi-\chi_{j}\right), \quad k=1,2, \cdots,(n-m) .
$$

From (6.3), (6.4), and (7.7), it may be seen that $\Omega_{k}\left(\chi_{i}\right)$ vanishes when $i \leqq k$, and when $i>n-m$, but that, on the contrary,

$$
\Omega_{k}\left(\chi_{i}\right) \neq 0, \quad \quad \text { for } i=k+1, k+2, \cdots, n-m .
$$

With any index $i$ from the set $1,2, \cdots, n-m$, and with $k=i-1$, the coefficients of the first $r$ powers of $1 / \lambda$ in the series within the brace of the formula (7.6) are the left-hand members of respective equations

$$
\begin{aligned}
\Omega_{i-1}\left(\chi_{i}\right) \alpha_{i, 0} & =1, \\
\Omega_{i-1}\left(\chi_{i}\right) \alpha_{i, j}+I\left(\alpha_{i, 0}, \cdots, \alpha_{i, j-1}\right) & =0, \quad j=1,2, \cdots, r-1 .
\end{aligned}
$$

Because of (7.8) we may determine the functions $\alpha_{i, j}$ successively for $j=0,1,2, \cdots, r-1$, to fulfill these equations, and we may do this for $i=1,2, \cdots, n-m$. Herewith the functions $u_{i}(z, \lambda)$ are determined.

The formulas (7.6) are now evidently of the forms

$$
\mathscr{D}_{k} L^{*}\left(u_{i}\right)=\lambda^{m+k} \exp \left(\lambda \int \sigma_{i} d z\right) B(z, \lambda),
$$

for every $i$ and $k$. By virtue of the relations (7.9), however, they are, more explicitly,

$$
\mathcal{D}_{k} L^{*}\left(u_{i}\right)=\lambda^{m+k} \exp \left(\lambda \int \sigma_{i} d z\right)\left\{1+\frac{B(z, \lambda)}{\lambda^{r}}\right\}, \quad \text { when } k=i-1,
$$


whereas it may be found from this that

$$
\mathscr{D}_{k} L^{*}\left(u_{i}\right)=\lambda^{m+k} \exp \left(\lambda \int \sigma_{i} d z\right) \frac{B(z, \lambda)}{\lambda^{r+1}}, \quad \text { when } k>i-1 .
$$

8. The related differential equation. Let the determinant $\Delta(u)$ be defined thus

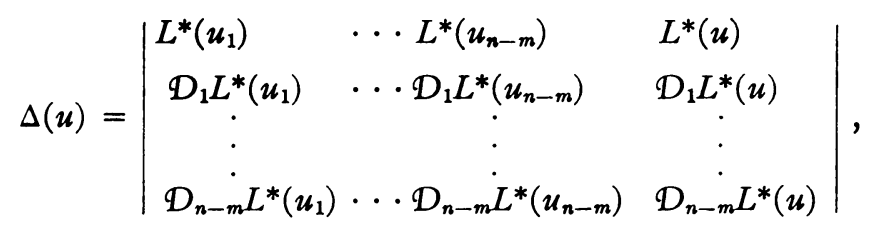

and let $\Delta_{i-1}$ signify the cofactor of the element in the $i$ th row and last column. Then with

$$
\mathcal{L}^{*}(u) \equiv \frac{\Delta(u)}{\Delta_{n-m}}
$$

the relation

$$
\mathcal{L}^{*}(u)=0
$$

is a linear differential equation of the order $n$, which admits as solutions the functions (7.1), as well as all solutions of the differential equation (6.5). For this latter equation let the solutions of any fundamental set (linearly independent) be denoted by $u_{n-m+1}(z, \lambda), \cdots, u_{n}(z, \lambda)$. These, as well as the functions (7.1), fulfill the equation (8.3). We wish to show that the members of this entire set are linearly independent.

The Wronskian $W$ of the functions $u_{i}(z, \lambda)$ for $i=1,2, \cdots, n$, is the determinant $\left|D^{i-1} u_{j}\right|$. By adding to each one of the last $(n-m)$ rows of this determinant a suitable linear combination of preceding rows, and observing that $\mathcal{D}_{k} L^{*}\left(u_{j}\right)$ vanishes for every $k$ when $j=n-m+1, \cdots, n$, because $L^{*}\left(u_{j}\right)$ is then identically zero, we see that

$(8.4)$

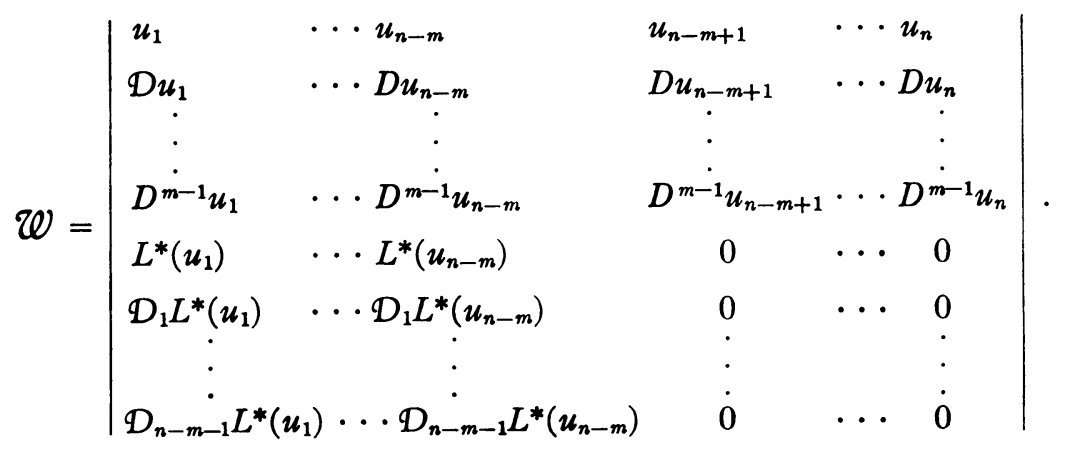


In this the $m$ rowed minor in the upper right-hand position is the nonvanishing Wronskian of the functions $u_{n-m+1}, \cdots, u_{n}$. The $(n-m)$ rowed minor in the lower left-hand position is $\Delta_{n-m}$, and is nonvanishing by virtue of the evaluations (7.10), (7.11), and (7.12). Thus $\mathscr{W} \neq 0$, and the functions $u_{i}(z, \lambda)$, $i=1,2, \cdots, n$, accordingly comprise a fundamental set of solutions of the differential equation (8.3). This equation is therefore solvable, in the sense that its solution forms are completely known.

The cofactor $\Delta_{n-m}$ has in its main diagonal elements of the form (7.11). Its elements below this diagonal, and above it, are respectively of the forms (7.12) and (7.10). The cofactor $\Delta_{i-1}$ is obtainable (except for sign) from $\Delta_{n-m}$ by replacing the elements of the latter's $i$ th row by the respective ones of the set $\mathcal{D}_{n-m} L^{*}\left(u_{j}\right), j=1,2, \cdots,(n-m)$. This replacement substitutes in the main diagonal the element $\mathcal{D}_{n-m} L^{*}\left(u_{i}\right)$ which is of the form

$$
\lambda^{n} \exp \left(\lambda \int \sigma_{i} d z\right) \frac{B(z, \lambda)}{\lambda^{r+1}},
$$

for the element $\mathscr{D}_{i-1} L^{*}\left(u_{i}\right)$ which is of the form

$$
\lambda^{m+i-1} \exp \left(\lambda \int \sigma_{i} d z\right)\left\{1+\frac{B(z, \lambda)}{\lambda^{r}}\right\} .
$$

This is the significant feature of the whole replacement. We see from it that

$$
\frac{\Delta_{i-1}}{\Delta_{n-m}}=\lambda^{n-m-i+1} \frac{B(z, \lambda)}{\lambda^{r+1}} .
$$

Now the expansion of the determinant $\Delta(u)$ by the elements of its last column yields the formula

$$
\mathcal{L}^{*}(u)=\mathscr{D}_{n-m} L^{*}(u)+\sum_{l=1}^{n-m} \frac{\Delta_{n-m-l}}{\Delta_{n-m}} \mathcal{D}_{n-m-l} L^{*}(u) .
$$

By (8.5), this has the form

$$
\mathcal{L}^{*}(u)=\mathcal{D}_{n-m} L^{*}(u)+\sum_{l=1}^{n-m} \lambda^{l} \frac{\mathcal{B}(z, \lambda)}{\lambda^{r+1}} \mathcal{D}_{n-m-l} L^{*}(u),
$$

and since

$$
\mathcal{D}_{n-m-l} L^{*}(u)=\sum_{j=0}^{n-l} \lambda^{i} B(z, \lambda) D^{n-j} u,
$$

it follows at once that

$$
\mathcal{L}^{*}(u)-\mathcal{D}_{n-m} L^{*}(u)=\frac{1}{\lambda^{r+1}} \sum_{i=1}^{n} \lambda^{i} B(z, \lambda) D^{n-i} u .
$$


This result may now be compared with the relation (6.6). Because of (6.7) the comparison shows that

$$
\mathcal{L}^{*}(u)=\mathcal{L}(u)+\sum_{i=1}^{n} \lambda^{i} \frac{B(z, \lambda)}{\lambda^{r+1}} D^{n-i} u,
$$

namely that the solvable equation (8.3) has coefficients which differ from those of the given differential equation (2.1) only by terms that are of at least the $(r+1)$ th degree in $1 / \lambda$. The differential equation (8.3) is therefore the related equation whose construction we proposed to accomplish.

\section{REFERENCES}

1. R. E. Langer, The boundary problem of an ordinary linear differential system in the complex domain, Trans. Amer. Math. Soc. vol. 46 (1939) pp. 151-190.

2. H. L. Turrittin, Asymptotic solutions of certain ordinary differential equations associated with multiple roots of the characteristic equation, Amer. J. Math. vol. 58 (1936) pp. 364-376.

3. R. E. Langer, The asymptotic solutions of ordinary linear differential equations of the second order, with special reference to a turning point, Trans. Amer. Math. Soc. vol. 67 (1949) pp. 461-490.

4. R. W. McKelvey, The solutions of second order linear ordinary differential equations about a turning point of order two, Trans. Amer. Math. Soc. vol. 79 (1955) pp. 103-123.

5. R. E. Langer, On the asymptotic forms of the solutions of ordinary linear differential equations of the third order in a region containing a turning point, Trans. Amer. Math. Soc. vol. 80 (1955) pp. 93-123.

6. - The solutions of a class of ordinary linear differential equations of the third order in a region containing a multiple turning point, to appear in Duke Math. J.

7. - A symptotic solutions of a differential equation in the theory of microwave propagation, Comm. on Pure and Applied Math. vol. 3 (1950) pp. 427-438.

8. W. Wasow, The complex asymptotic theory of a fourth order differential equation of hydrodynamics, Ann. of Math. vol. 49 (1948) pp. 852-871.

9. - A study of the solutions of the differential equation $y^{\prime \prime \prime \prime}+\lambda^{2}\left(x y^{\prime \prime}+y\right)=0$, for large values of $\lambda$, Ann. of Math. vol. 52 (1950) pp. 350-361.

10. D. Meksyn, Asymptotic integrals of a fourth order differential equation containing a large parameter, Proc. London Math. Soc. (2) vol. 49 (1947) pp. 436-457.

11. - Stability of viscous flow between rotating cylinders, Proc. Royal Soc. London Ser. A. vol. 187 (1946) pp. 115-128 and 480-504.

UNIVERSITY OF WISCONSIN, Madison, Wis. 